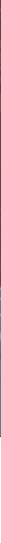

\title{
Niveau de formation et investissement dans l'éducation dans les pays ibéro-américains
}

- Dans tous les pays ibéro-américains couverts par Regards sur l'éducation, les adultes sont moins susceptibles d'être diplômés du deuxième cycle du secondaire que la moyenne de l'OCDE, même si l'écart est moindre chez les plus jeunes.

- Dans les pays de l'OCDE, le pourcentage de diplômés de l'enseignement tertiaire est sensiblement plus élevé chez les 25-34 ans que chez les 55-64 ans. Ce constat vaut aussi pour les pays ibéro-américains, mais la hausse n'est supérieure à la moyenne de l'OCDE qu'en Espagne et au Portugal, tandis que les autres pays de ce groupe restent en retrait.

- Les gouvernements ibéro-américains font face à une demande croissante dans le domaine de l'éducation. Au Chili, en Colombie, au Costa Rica et au Portugal, la part des dépenses privées au titre des établissements d'enseignement tertiaire est supérieure à la moyenne de l'OCDE, en pourcentage du PIB.

Encadré 1. Regards sur l'éducation et pays ibéro-américains couverts dans son édition 2016

Publication de référence sur l'état de l'éducation dans le monde, Regards sur l'éducation : Les indicateurs de l'OCDE fournit des données clés sur la structure, le financement et la performance des systèmes d'éducation des 35 pays de l'OCDE, ainsi que d'un certain nombre de pays partenaires. Ce numéro des Indicateurs de l'éducation à la loupe s'intéresse plus particulièrement aux pays ibéro-américains couverts dans l'édition 2016 de Regards sur l'éducation.

Par pays ibéro-américains, on entend le Brésil, l'Espagne, le Portugal et l'ensemble des pays hispanophones d'Amérique du Nord, d'Amérique centrale et d'Amérique du Sud.

L'édition 2016 de Regards sur l'éducation présente des données pour les pays ibéro-américains suivants :

- Pays européens : Espagne et Portugal.

- Pays latino-américains membres de l'OCDE : Chili et Mexique.

- Pays latino-américains candidats à l'adhésion : Colombie et Costa Rica.

- Pays latino-américains partenaires : Argentine et Brésil.

Dans les pays ibéro-américains, le niveau de formation est très différent de la moyenne de l'OCDE

Dans les pays de l'OCDE, 16 \% des 25-34 ans ne sont pas diplômés du deuxième cycle du secondaire, 42 \% sont au plus diplômés du deuxième cycle du secondaire et $42 \%$ sont diplômés de l'enseignement tertiaire. Dans tous les pays ibéro-américains, la répartition des jeunes adultes entre les niveaux de formation est très différente - le pourcentage de non-diplômés du deuxième cycle du secondaire y est en particulier bien plus élevé, et celui des diplômés de l'enseignement tertiaire, bien plus faible (graphique 1).

Graphique 1. Pourcentage de 25-34 ans selon le niveau de formation (2015)

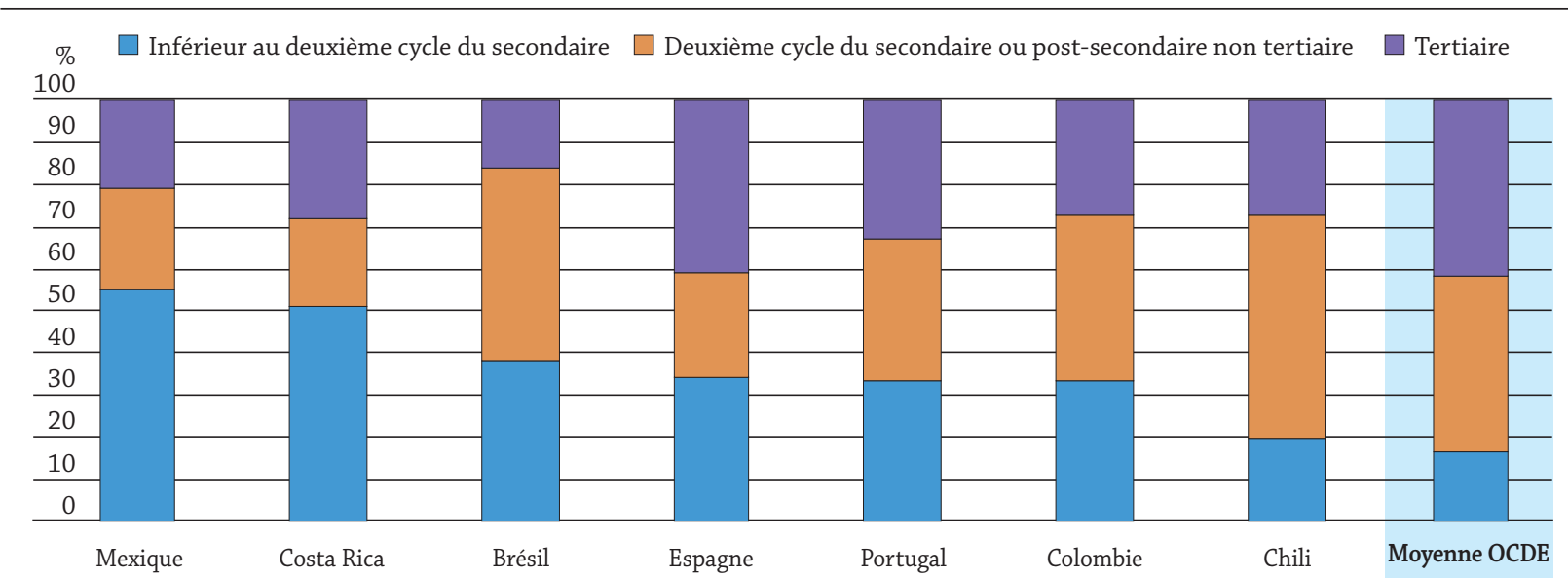

Remarque : Année de référence : 2014 pour le Brésil, et 2013 pour le Chili.

Les pays sont classés par ordre décroissant du pourcentage de jeunes adultes dont le niveau de formation est inférieur au deuxième cycle du secondaire.

Source : OCDE, Regards sur l'éducation (base de données), http://stats.oecd.org. 
Un pourcentage élevé de jeunes adultes non diplômés du deuxième cycle du secondaire

Le diplôme du deuxième cycle du secondaire représente le niveau minimum de formation nécessaire à la réussite de l'entrée sur le marché du travail et à la pérennité de l'employabilité. Dans les pays de l'OCDE, les adultes non diplômés de ce niveau d'enseignement présentent les taux les plus élevés de chômage et d'inactivité, ainsi que des revenus inférieurs et diminuant plus rapidement au cours de leur vie professionnelle, en particulier durant les années précédant leur départ à la retraite. Un pourcentage élevé d'actifs occupés peu qualifiés peut ainsi induire d'importantes dépenses publiques de sécurité sociale et entrainer le creusement d'inégalités à la fois difficiles et coûteuses à enrayer une fois les individus déscolarisés.

Ces dernières décennies, le pourcentage d'adultes non diplômés du deuxième cycle du secondaire a diminué dans la majorité des pays membres ou partenaires de l'OCDE, pour s'établir en 2015 à 23 \% parmi les 25-64 ans, en moyenne, dans les pays de l'OCDE. D'après les données de l'édition 2016 de Regards sur l'éducation, les pays ibéro-américains accusent du retard sur cette tendance, avec des pourcentages élevés d'adultes non diplômés de ce niveau d'enseignement : environ $60 \%$ au Costa Rica et au Mexique ; environ $50 \%$ au Brésil, en Colombie et au Portugal ; et environ $40 \%$ au Chili et en Espagne. En outre, comme nous le rappelle le Programme international pour le suivi des acquis des élèves (PISA), l'élargissement de l'accès à l'enseignement secondaire ne se traduit pas systématiquement par une amélioration des résultats d'apprentissage.

Un pourcentage plus important de jeunes adultes sont diplômés du deuxième cycle du secondaire dans les pays ibéro-américains. Les écarts entre les générations sont plus marqués dans les pays ibéro-américains que dans la zone OCDE considérée dans son ensemble, en partie parce que le pourcentage d'adultes non diplômés du deuxième cycle du secondaire est sensiblement plus élevé chez les adultes plus âgés. C'est au Chili, en Colombie et au Portugal que l'augmentation du pourcentage de diplômés du deuxième cycle du secondaire est la plus forte entre les générations. II est intéressant de noter qu'au Chili, le pourcentage de jeunes adultes non diplômés du deuxième cycle du secondaire est similaire à la moyenne de l'OCDE. À l'autre extrémité du spectre, les progrès sont plus lents au Costa Rica et au Mexique, où plus de la moitié des jeunes adultes ne sont toujours pas diplômés du deuxième cycle du secondaire (graphique 2).

\section{Graphique 2. Pourcentage d'adultes non diplômés du deuxième cycle du secondaire,} selon le groupe d'âge (2015)

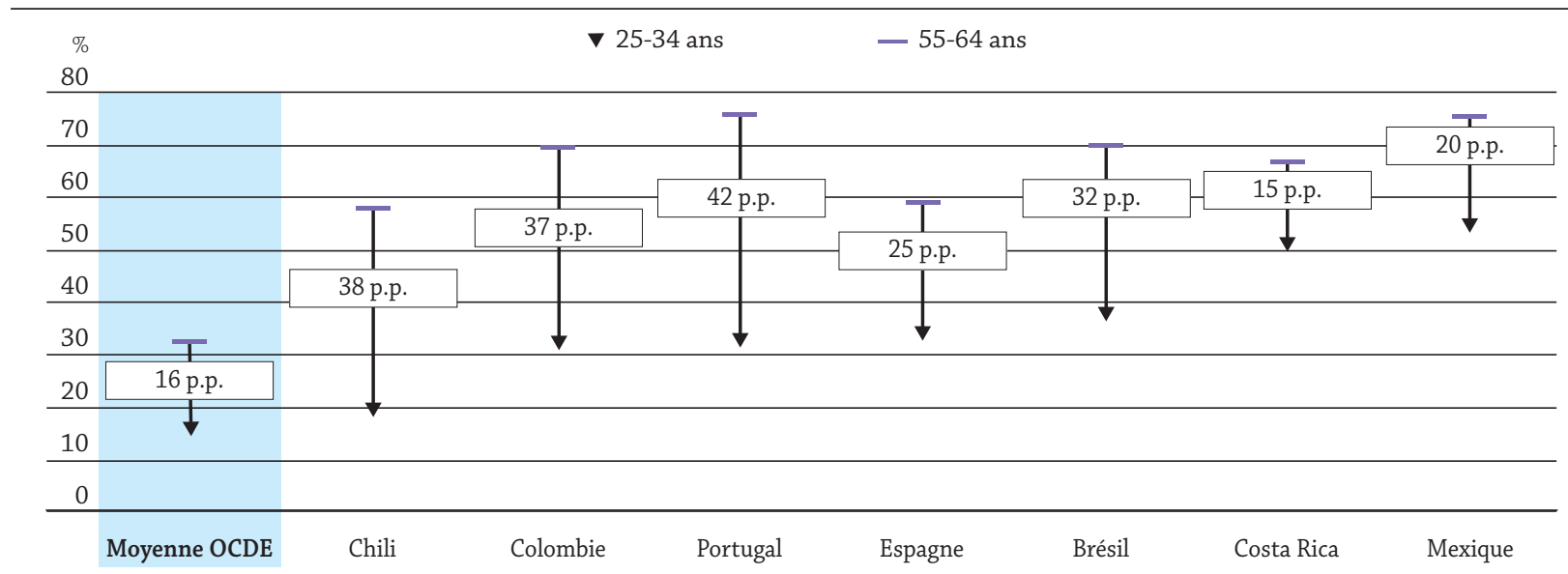

Remarque : Année de référence : 2014 pour le Brésil, et 2013 pour le Chili. Les valeurs indiquées dans le graphique représentent la différence (exprimée en points de pourcentage [p.p.]) de pourcentage d’adultes non diplômés du deuxième cycle du secondaire entre les plus âgés et les plus jeunes. Les pays sont classés par ordre croissant du pourcentage de 25-34 ans non diplômés du deuxième cycle du secondaire. Source : OCDE, Regards sur l'éducation (base de données), http://stats.oecd.org.

\section{La situation d'ensemble s'améliore avec l'augmentation de la demande d'éducation, notamment dans l'enseignement tertiaire}

L'enseignement tertiaire a aussi connu une belle expansion, bien que la plupart des pays ibéro-américains restent largement en retrait par rapport à la moyenne de l'OCDE. Le pourcentage de jeunes adultes diplômés de l'enseignement tertiaire a connu une progression constante dans les pays de l'OCDE. Parmi les pays ibéro-américains, c'est en Espagne et au Portugal que l'augmentation a été la plus forte : le pourcentage de diplômés de l'enseignement tertiaire y est désormais supérieur d'environ 20 points de pourcentage chez les 25-34 ans que chez les 55-64 ans. Dans le reste des pays ibéro-américains couverts, la progression a été inférieure à celle de 16 points de pourcentage observée en moyenne dans les pays de l'OCDE. Au Brésil, au Costa Rica et au Mexique, le pourcentage de diplômés de l'enseignement tertiaire a notamment augmenté de moins de 10 points de pourcentage entre ces deux générations. Si de bons indicateurs pour l'évaluation de la qualité de l'enseignement tertiaire et des résultats d'apprentissage des étudiants et des diplômés restent à développer, les données de l'Évaluation des compétences des adultes, lancée dans le cadre du Programme pour l'évaluation internationale des compétences des adultes (PIAAC), semblent néanmoins indiquer qu'un diplôme de l'enseignement tertiaire ne garantit pas nécessairement l'équivalence du niveau de compétence, et que ce dernier varie sensiblement chez les diplômés, tant entre les pays qu'au sein de ceux-ci.

L'Espagne est le seul pays ibéro-américain où le pourcentage de jeunes adultes diplômés de l'enseignement tertiaire est similaire à la moyenne de l'OCDE (41 \% et $42 \%$, respectivement). Au Portugal, un jeune adulte sur trois est diplômé de l'enseignement tertiaire, et dans le reste des pays de ce groupe, ils sont moins de $30 \%$, les pourcentages les plus faibles s'observant au Brésil (16 \%) et au Mexique (21 \%).

L'augmentation du nombre de diplômés de l'enseignement tertiaire est encore plus marquée parmi les femmes, celles des générations précédentes étant moins susceptibles que les hommes d'avoir obtenu un diplôme de ce niveau d'enseignement. Dans les pays ibéro-américains comme dans les pays de 


\section{INDICATEURS DE L'ÉDUCATION À LA LOUPE}

I'OCDE, le pourcentage de diplômés de l'enseignement tertiaire est plus élevé chez les jeunes femmes que chez les jeunes hommes. Toutefois, même si les jeunes femmes des pays ibéro-américains devancent leurs pairs de sexe masculin, elles restent plus éloignées de la moyenne de l'OCDE que ces derniers, sauf au Portugal. Ce constat vaut particulièrement pour les jeunes femmes du Chili et du Mexique, qui accusent un écart avec leurs pairs de I'OCDE au moins deux fois plus important que celui observé pour les jeunes hommes.

Dans la vie d'un individu, l'éducation est associée à un certain nombre de retombées sociales et économiques. II existe ainsi une relation positive entre le niveau de formation et des retombées sociales telles que l'état de santé, le bénévolat, la confiance interpersonnelle et l'efficacité politique. En outre, les adultes diplômés de l'enseignement tertiaire bénéficient sur le marché du travail de revenus supérieurs à ceux des adultes diplômés au plus du deuxième cycle du secondaire. En moyenne, dans les pays de I'OCDE, les adultes diplômés de l'enseignement tertiaire travaillant à temps plein ont des revenus supérieurs d'environ 55 \% à ceux des adultes diplômés du deuxième cycle du secondaire. Parmi tous les pays membres ou partenaires de l'OCDE, ce sont quatre pays ibéro-américains qui présentent l'avantage financier le plus important en faveur des adultes diplômés de l'enseignement tertiaire : le Brésil (141\%), le Chili (139\%), la Colombie (133\%) et le Mexique (105\%).

\section{La contribution des ménages à l'enseignement obligatoire est élevée}

Chacun sait que l'élévation du niveau de formation nécessite de consentir des investissements substantiels dans l'éducation. Dans les pays ibéro-américains, les dépenses publiques et privées au titre de l'éducation restent relativement faibles en valeur absolue, mais représentent une part comparativement importante du produit intérieur brut (PIB).

Comme en général dans les pays de l'OCDE, la plupart des dépenses au titre de l'enseignement obligatoire proviennent de sources publiques dans les pays ibéro-américains, mais les dépenses privées représentent une part plus importante des dépenses totales qu'en moyenne dans les pays de l'OCDE. C'est en Colombie que la part des dépenses privées est la plus élevée, s'établissant à $1.0 \%$ du PIB, et en Espagne qu'elle est la plus faible, à $0.4 \%$. Au Chili, les dépenses privées représentent le double de la moyenne de l'OCDE $(0.7 \%$ et $0.3 \%$, respectivement), mais les dépenses totales restent inférieures à cette moyenne (3.1 \% et $3.6 \%$, respectivement). Ces chiffres soulignent la plus grande importance des sources privées dans le financement de l'enseignement obligatoire, et donc de l'élévation du niveau de formation, dans les pays ibéro-américains que dans d'autres pays de l'OCDE.

\section{L'accroissement de la demande dans l'enseignement supérieur est financé par une combinaison de sources} publiques et privées

L'augmentation de la participation à l'enseignement tertiaire dans les pays ibéro-américains s'est aussi accompagnée de la hausse des dépenses privées d'éducation. Comme maints autres pays de l'OCDE, de nombreux gouvernements ibéro-américains éprouvent des difficultés à dégager les ressources nécessaires pour répondre à la demande croissante d'éducation par le seul recours aux financements publics.

En général, dans les pays ibéro-américains, la part des dépenses privées au titre des établissements d'enseignement tertiaire, exprimée en pourcentage du PIB, est supérieure à la moyenne de l'OCDE (0.5\%). Cette part s'établit à $1.4 \%$ au Chili, à $1.1 \%$ en Colombie, à $1.0 \%$ au Costa Rica et à $0.5 \%$ au Portugal.

La plus grande nécessité de recourir à des sources privées de financement pour faire des études supérieures peut limiter l'accès à l'enseignement tertiaire. Dans les pays ibéro-américains où la part des dépenses privées en pourcentage du PIB est plus élevée que la moyenne de l'OCDE, le pourcentage de diplômés de l'enseignement tertiaire tend à être plus faible, sauf au Mexique. Le Chili, la Colombie et le Costa Rica, où la part des dépenses privées au titre de l'enseignement tertiaire est relativement importante, présentent les pourcentages les plus faibles de jeunes adultes diplômés de l'enseignement tertiaire (inférieurs d'environ 14 points de pourcentage à la moyenne de l'OCDE). En revanche, des niveaux plus faibles de financement privé, comme ceux observés en Espagne et au Portugal, sont associés à des pourcentages plus élevés de diplômés de l'enseignement tertiaire (graphique 3).

\section{Graphique 3. Dépenses privées au titre des établissements d'enseignement en pourcentage du PIB (2013) et pourcentage de jeunes adultes diplômés de l'enseignement tertiaire (2015)}

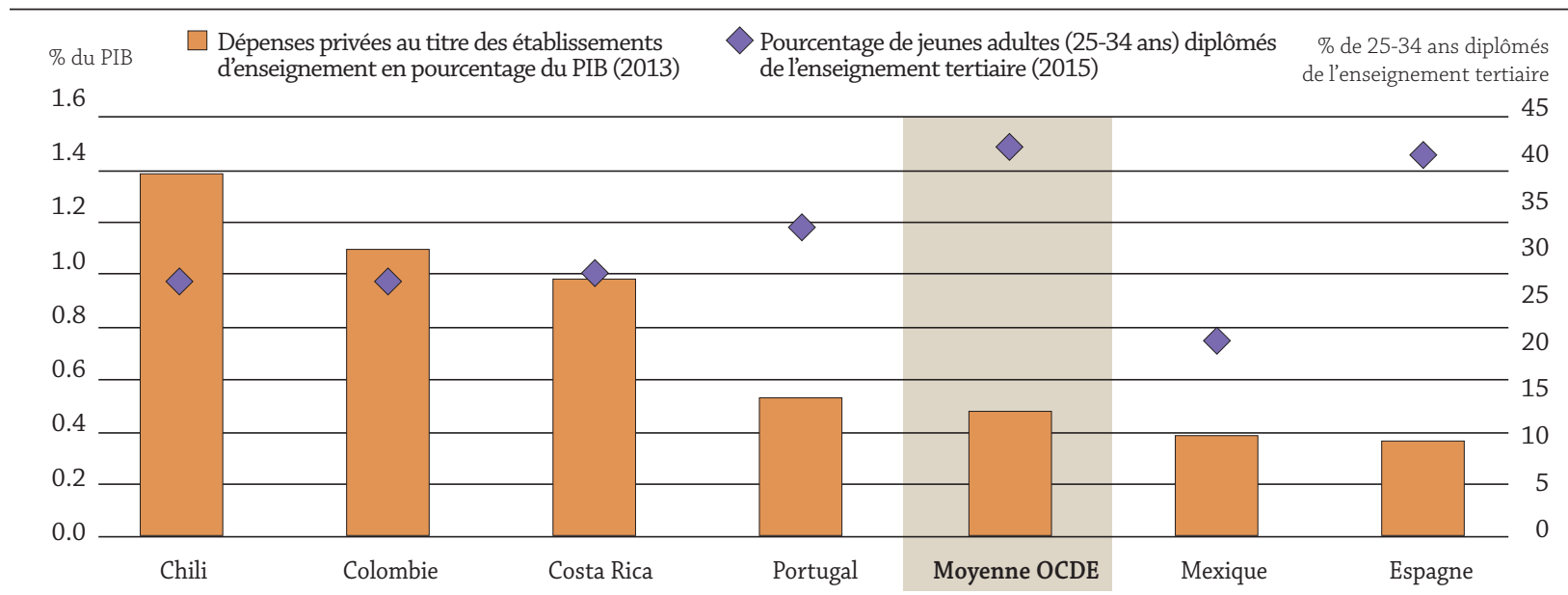

Remarque : Les données du Chili se rapportent à 2014 et n'incluent pas les sources internationales. Le financement privé inclut les dépenses consenties par les élèves/étudiants, les ménages et d’autres entités privées.

Les pays sont classés par ordre décroissant des dépenses privées en pourcentage du PIB.

Source : OCDE, Regards sur l'éducation (base de données), http://stats.oecd.org. 
Dans les pays latino-américains, l'augmentation des dépenses d'éducation peut avoir une incidence positive sur la performance en sciences dans l'enquête PISA

Le Programme international pour le suivi des acquis des élèves (PISA) évalue la mesure dans laquelle les élèves de 15 ans ont acquis, alors qu'ils approchent de la fin de leur scolarité obligatoire, les connaissances et compétences clés nécessaires à leur pleine participation à nos sociétés modernes. La dernière évaluation, administrée en 2015 dans 73 pays et économies, met en évidence l'existence d'une relation positive entre l'augmentation des dépenses d'éducation et la performance en sciences, et ce jusqu'à un seuil moyen de dépenses de 50000 USD par élève (graphique 4). Le montant moyen des dépenses par élève entre l'âge de 6 et 15 ans indique l'effet cumulatif que les dépenses peuvent avoir sur les résultats d'apprentissage d'un élève au cours de sa scolarité. Dans la plupart des pays ibéro-américains, le montant des dépenses par élève est inférieur au seuil de 50000 USD, ce qui semble indiquer que l'augmentation du niveau des dépenses pourrait renforcer la capacité de ces pays à offrir à tous leurs élèves une éducation de qualité

\section{Graphique 4. Dépenses par élève entre l'âge de 6 et 15 ans (2013) et performance en sciences dans l'enquête PISA (2015)}

$\square$ Pays/économies dont les dépenses cumulées par élève étaient inférieures à 50 000 USD en 2013
$\square$ Pays/économies dont les dépenses cumulées par élève étaient égales ou supérieures à 50 000 USD en 2013

Remarque : Seuls sont nommés dans le graphique les pays ibéro-américains couverts dans l'édition 2016 de Regards sur l'éducation, mais tous les pays et économies disposant de données dans la base de données PISA 2015 sont utilisés pour le calcul des lignes de régression. La ligne en maigre indique une relation significative $(\mathrm{p}<0.10)$, et celle en gras, une relation non significative $(\mathrm{p}>0.10)$.

Source : OCDE, Résultats du PISA 2015, Base de données du Volume II, http://dx.doi.org/10.1787/888933436215, graphique II.6.2.

Pour conclure : Malgré la distance géographique qui les sépare, les pays ibéro-américains présentent certaines similitudes, notamment concernant le niveau de formation et la part des dépenses privées au titre des établissements d'enseignement en pourcentage du PIB. Dans tous les pays ibéro-américains couverts par Regards sur l'éducation, le pourcentage d’adultes non diplômés du deuxième cycle du secondaire est supérieur à la moyenne. En outre, même si l'écart se réduit dans la jeune génération, le pourcentage d’adultes moins instruits y reste supérieur à la moyenne de l'OCDE. Parallèlement, la part des dépenses privées au titre des établissements d'enseignement en pourcentage du PIB est en général plus élevée dans les pays ibéro-américains que la moyenne de l'OCDE. Ces caractéristiques expliquent en partie les inégalités socio-économiques prévalant dans les pays ibéro-américains, ceux ayant les moyens de payer leurs études (ou celles de leurs enfants) accédant à de meilleurs emplois et revenus, et se trouvant ainsi en mesure de progresser sur l'échelle sociale.

\section{Pour tout complément d'information}

- OCDE (2016a), Regards sur l'éducation 2016 : Les indicateurs de l'OCDE, Éditions OCDE, Paris, http://dx.doi.org/10.1787/eag-2016-fr.

- OCDE (2016b), Résultats du PISA 2015 (Volume II) : Politiques et pratiques pour des établissements performants, Éditions OCDE, Paris, http://dx.doi. org/10.1787/9789264267558-fr.

- OCDE (2016c), L’importance des compétences : Nouveaux résultats de l'évaluation des compétences des adultes, Éditions OCDE, Paris, http://dx.doi. org/10.1787/9789264259492-fr.

Les données et d'autres types de ventilations peuvent être consultés sur http://stats.oecd.org/, Regards sur l'éducation (base de données).

\section{Contacter}

Gara Rojas González (Gara.RojasGonzalez@oecd.org) et Daniel Sánchez Serra (Daniel.SanchezSerra@oecd.org)

\begin{tabular}{l|l}
$\begin{array}{l}\text { Voir } \\
\text { www.oecd.org/fr/education/regards-sur-l-education-19991495.htm }\end{array}$ & $\begin{array}{l}\text { Prochain numéro } \\
\text { Réforme des frais de scolarité et mobilité internationale }\end{array}$ \\
$\begin{array}{l}\text { Les indicateurs de l'éducation à la loupe (numéros précédents) } \\
\text { PISA à la loupe }\end{array}$ & \\
\hline L'enseignement à la loupe & \\
\hline
\end{tabular}

Crédit photo : () Ghislain \& Marie David de Lossy/Cultura/Getty Images

Ce document est publié sous la responsabilité du Secrétaire général de I'OCDE. Les opinions qui y sont exprimées et les arguments qui y sont employés ne reflètent pas nécessairement les vues officielles des pays membres de l'OCDE.

Ce document, ainsi que les données et cartes qu'il peut comprendre, sont sans préjudice du statut de tout territoire, de la souveraineté s'exerçant sur ce dernier, du tracé des frontières et limites internationales, et du nom de tout territoire, ville ou région.

Les données statistiques concernant Israël sont fournies par et sous la responsabilité des autorités israéliennes compétentes. L'utilisation de ces données par l'OCDE est sans préjudice du statut des hauteurs du Golan, de Jérusalem-Est et des colonies de peuplement israéliennes en Cisjordanie aux termes du droit international. 